\title{
Multiperiodic RR Lyrae stars in the Galactic Bulge and the SMC
}

\author{
Tomasz Mizerski \\ Warsaw University Observatory, Al. Ujazdowskie 4, 00-478 Warszawa, \\ Poland
}

\begin{abstract}
I have performed a detailed analysis of multiperiodic RR Lyr stars of the Galactic Bulge and the Small Magellanic Cloud. In the rich OGLE-II database on the Galactic Bulge objects I detected more than 2500 RR Lyr stars, with almost 600 of them exhibiting multiperiodic behavior of various, sometimes unique, kinds. Many of them can only be explained by excitation of nonradial modes. There are two major, striking differences between the two discussed stellar systems: the incidence rate of RRd double mode pulsators is over 30 times higher in the SMC than in the Galactic Bulge, and there are more than twice as many Blazhko stars in the Galactic Bulge as in the SMC.
\end{abstract}

\section{Introduction}

RR Lyr stars play an important role in modern astrophysics. They are widely used, but not fully understood. For many years these stars were thought to be very simple stars, purely radial pulsators. In recent years, however, evidence for nonradial oscillations in RR Lyr stars has been found. I have undertaken a detailed analysis of RR Lyr stars in the Galactic Bulge and the SMC to investigate all multiperiodic stars, especially those with nonradial modes excited.

\section{Double mode radial pulsators}

Double mode pulsators, in which the two first radial modes are excited simultaneously, are very easy targets for detection. The first such star was identified by Jerzykiewicz \& Wentzel (1977). RR Lyr stars of this type are called RRd stars. My search for RRd variables in the OGLE-II database gave very intriguing results. I found only $3 \mathrm{RRd}$ stars among more than $2700 \mathrm{RR}$ Lyr stars in the Galactic Bulge. In the SMC, where I analyzed only about 600 RR Lyr stars, 61 RRd stars were found. This is a very significant fact: RRd stars are much more numerous in the SMC than in the Galactic Bulge, where they are almost completely absent. Alcock et al. (1996) also report high percentage of RRd stars in the LMC. Properties of RRd stars in the SMC are very similar to those given by Kovács (1999). The most widely used parameter, the ratio of the frequencies of the radial modes, is in the range 0.742 to 0.747 , with an average value of 0.745 . As in the LMC, the first overtone is the dominant mode in most cases. 
Characteristics of the three RRd stars from the Galactic Bulge are given in Table 1 , where $P_{1} / P_{0}$ is the period ratio, and $A_{0} / A_{1}$ is the amplitude ratio.

Table 1. RRd stars in the Galactic Bulge.

\begin{tabular}{ccccc}
\hline star & $P_{0}$ & $P_{1}$ & $P_{1} / P_{0}$ & $A_{0} / A_{1}$ \\
\hline bul 7.1529 & 0.487192 & 0.362842 & 0.744761 & 0.464 \\
bul21.7133 & 0.503068 & 0.374970 & 0.745366 & 0.176 \\
bul 39.1568 & 0.461390 & 0.344906 & 0.747537 & 0.334 \\
\hline \hline
\end{tabular}

\section{Blazhko stars}

Blazhko stars have been known for almost a century, but their nature is not yet fully understood. In the recent literature they are divided into two classes (Moskalik \& Poretti (2002): BL1 and BL2 Blazhko stars. BL1 stars have one additional peak in the periodogram, located in the vicinity of the peak corresponding to the radial mode of pulsation. In periodograms of BL2 stars two additional peaks are present placed symmetrically on both sides of the peak of the radial mode. Together with the radial mode they form an equidistant frequency triplet. Examples of BL1 and BL2 stars are shown in Fig. 1. The top panel contains a purely radial pulsator for comparison.

The presence of frequency triplets could be explained without involving nonradial oscillations, but the existence of doublets in BL1 does not leave any other choices. BL1 stars are therefore considered examples of nonradial pulsators. In those stars at least one nonradial mode, with a frequency similar to the frequency of the radial mode, is excited.

There are many Blazhko stars among RRab stars. I discovered $243 \mathrm{BL} 1$ and 143 BL2 RRab stars in the Galactic Bulge. If we define $\Delta f$ as the frequency separation between the additional peak and the radial mode, then $\Delta f$ is positive in about $75 \%$ of RRab Blazhko stars. This means that the additional peak in the case of BL1, and the higher of the two additional peaks in the case of BL2, is on the right side of the radial mode and has a higher frequency. Triplets range from highly symmetric to highly asymmetric. Modulation periods for RRab BL stars are on the order of $100 \mathrm{~d}$. This is not the case with RRc BL variables. Their modulation periods are about twice as long and in most cases $\Delta f$ is negative. The additional peak is also higher compared to the amplitude of the radial mode. I found 22 RRc BL1 and 30 RRc BL2 variables in the Galactic Bulge. Unlike the RRab stars, BL1 stars are less numerous than BL2 stars among RRc stars. The reason for the differences between RRab and RRc Blazhko stars is unknown.

In the SMC the total number of Blazhko stars is 65 , which is about $10 \%$ of all RR Lyr stars in that system. As in the Galactic Bulge, RRab BL1 stars were found to be more numerous than RRab BL2 stars.

There are also a few stars in both systems that seem to be BL1 stars with modulation periods longer than the current time span of the data. Further observations are needed to confirm this hypothesis. 


\section{Stars with multiple periods}

During the analysis of OGLE-II data I discovered RRLyr stars that closely resemble Blazhko variables but could not be classified as either BL1 or BL2. Periodograms of these stars reveal the presence of more than two additional high peaks in the vicinity of the radial mode. Because of their similarity to Blazhko stars I will call them BL3. If these stars were to be considered Blazhko variables, then the total number of Blazhko stars in the Galactic Bulge would reach 600 . That would amount to $22 \%$ of all RR Lyr stars. There are 86 BL3 stars among RRab stars, and 41 BL3 stars among RRc stars.

BL3 stars are also examples of nonradial pulsators. Additional peaks can not correspond to radial modes.

\section{Summary}

I presented here the results of the analysis of RR Lyr stars in two very different stellar systems: the Galactic Bulge and the Small Magellanic Cloud. The picture that emerges from my investigation is that while the Galactic Bulge is very rich in Blazhko stars (about 20\%) and very poor in RRd stars, the situation in the SMC is exactly the opposite. It is relatively poor in Blazhko variables (about $10 \%$ ) and very rich in RRd stars. In fact RRd stars are proportionally 30 times more numerous in the SMC than in the Galactic Bulge. The LMC seems to be somewhere inbetween. These two significant differences are not understood and call for an explanation.

Acknowledgments. I would like to thank Dr Wojciech Dziembowski for many inspiring discussions. This work was supported by Polish KBN grant 5 P03D 03020.

\section{References}

Alcock, C. et al. 1996, AJ, 111, 1146

Jerzykiewicz, M., Wentzel, W. 1977, Acta Astron., 27, 35

Kovács, G. 1999, ApJ., 512, 271

Moskalik, P., Poretti, E. 2002, in ASP Conf. Ser., Vol. 259, Radial and Nonradial Pulsations as Probes of Stellar Physics, eds C. Aerts, T.R. Bedding \& J. Christensen-Dalsgaard (San Francisco: ASP), 392 

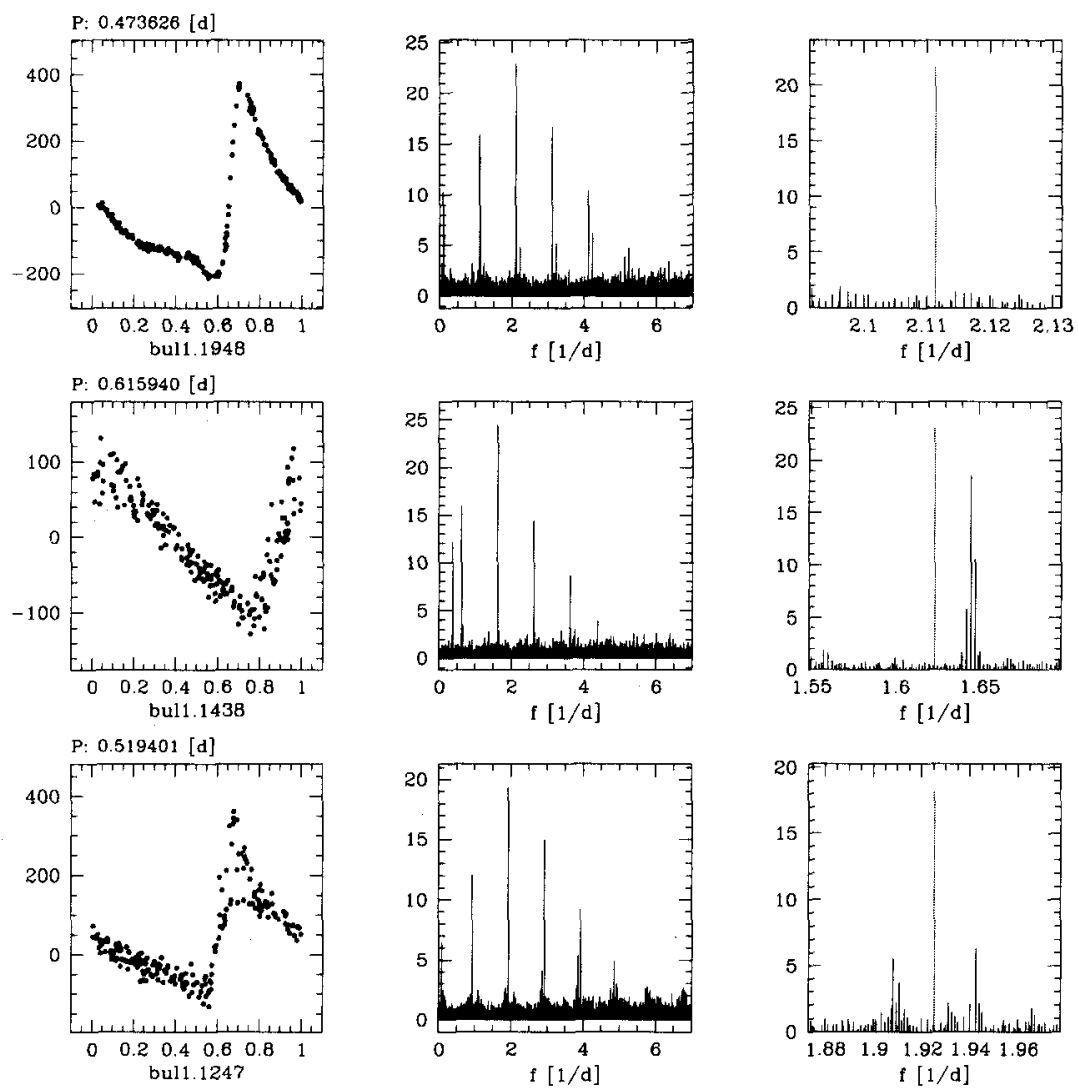

Figure 1. RRab Blazhko stars. Each panel contains a phased light curve and Lomb-Scargle periodograms before and after subtraction of the dominant frequency, that is the frequency of the radial mode. The line in the center of every prewhitened periodogram marks the location of the subtracted frequency. 